\title{
Serum Anti-Müllerian Hormone Levels in Patients with Epithelial Ovarian Cancer
}

\author{
Pawel Walentowicz, ${ }^{1}$ Pawel Sadlecki, ${ }^{1}$ Magdalena Krintus, ${ }^{2}$ Grazyna Sypniewska, ${ }^{2}$ \\ Aneta Mankowska-Cyl, ${ }^{2}$ Marek Grabiec, ${ }^{1}$ and Malgorzata Walentowicz-Sadlecka ${ }^{1}$ \\ ${ }^{1}$ Department of Obstetrics and Gynecology, L. Rydygier Collegium Medicum in Bydgoszcz, Nicolaus Copernicus University, \\ Ulica Ujejskiego 75, 85-168 Bydgoszcz, Poland \\ ${ }^{2}$ Department of Laboratory Medicine, L. Rydygier Collegium Medicum in Bydgoszcz, Nicolaus Copernicus University, \\ Ulica Jurasza 5, 85-094 Bydgoszcz, Poland
}

Correspondence should be addressed to Malgorzata Walentowicz-Sadlecka; walentowiczm@cm.umk.pl

Received 10 May 2013; Revised 28 June 2013; Accepted 5 July 2013

Academic Editor: Kai J. Buhling

Copyright (C) 2013 Pawel Walentowicz et al. This is an open access article distributed under the Creative Commons Attribution License, which permits unrestricted use, distribution, and reproduction in any medium, provided the original work is properly cited.

Objectives. The aim of our study was to examine serum anti-Müllerian hormone (AMH) concentration in ovarian cancer patients in relation to clinicopathological features, such as a pathological subtype of the tumor, (FIGO) stage, grading, and overall 5-year survival. Material and Methods. We enrolled 72 epithelial ovarian cancer patients in our study, aged 45-79 years, who underwent optimal cytoreductive surgery. In all patients, serum AMH concentration was measured using a two-step sandwich type enzyme immunoassay before surgery. As a reference value for women over 45 years we accepted anti-Müllerian hormone concentration below $1 \mathrm{ng} / \mathrm{mL}$. Results. In the whole group of patients with ovarian cancer, median serum concentration of AMH was 0.07 (0.0$0.37) \mathrm{ng} / \mathrm{mL}$, whereas in the group of those with positive AMH values $(\geq 0.14 \mathrm{ng} / \mathrm{mL})$ it was $0.31(0.15-0.73) \mathrm{ng} / \mathrm{mL}$. No significant correlation was found between serum AMH levels and FIGO stage, histological subtype, or grading $(P>0.05)$. The analysis of five-year survival rate related to AMH levels showed no statistically significant differences. There were no differences in survival rates between patients with positive or negative serum AMH levels. Conclusion. Measurement of serum anti-Müllerian hormone levels was not useful in predicting clinicopathological features and survival in patients with ovarian cancer.

\section{Introduction}

Among cancers of female reproductive system, ovarian cancer became the second most common and is the fifth ranked cause of cancer-related mortalities in women in Europe and the United States [1]. Although understanding of ovarian cancer has improved substantially, the etiology of the disease remains unknown. Moreover, even though surgical procedures made a great progress and new protocols of chemotherapy were introduced, the 5-year survival rate does not exceed $45 \%$. The main reason for this situation is that the majority of women with ovarian cancer turn to gynecologist for help at the late stage of the disease and that despite introducing new markers for the detection of ovarian cancer, their low diagnostic sensitivity does not permit to use them as screening $[2,3]$.
Anti-Müllerian hormone (AMH), also known as Müllerian inhibiting substance (MIS), belongs to a larger family of transforming growth factor- $\beta$ (TGF- $\beta$ ). AMH signals through two transmembrane receptors, type II which is specific (present in Müllerian duct and gonads) and type I receptors, shared with the bone morphogenetic proteins family $[4,5]$.

AMH is expressed in the Sertoli cells of fetal testis from the seventh week of pregnancy [6], and its secretion is fundamental in regression of the Müllerian ducts [7].

In women AMH is produced by granulosa cells, from preantral and antral follicles. Serum AMH levels are undetectable in newborns, increase during childhood and adolescence reaching its peak in the early 20 s, and remain stable throughout reproductive period only to decrease during menopausal transition $[8,9]$. During menstrual cycle serum 
AMH maintains its level and lowers slightly in early secretory phase $[10,11]$.

Studies on mice had shown that AMH inhibits the transition from the primordial to the primary follicular stage. AMH paracrine signaling inhibits FSH-related follicle growth leading to selection of primary follicle. FSH and estradiol decrease AMH gene expression in granulosa cells [12]. Recent in vitro studies confirmed that increased expression of AMH is caused by bone morphogenetic protein (BMP-6) and also that $\mathrm{AMH}$ inhibits recruitment of primordial follicles in order to keep ovarian function in balance [13].

The number of primordial follicles decreases with age of women. Lower serum AMH levels precede the increase of FSH and inhibin B levels and thus are the most accurate parameter of ovarian reserve in clinical practice [1416]. AMH possesses high prognostic value in prediction of number of obtained oocytes [17, 18]. Besides, ovarian hyperstimulation syndrome seems to be associated with significantly higher basal AMH levels [19].

In women undergoing oncologic treatment $\mathrm{AMH}$ is considered a useful marker of damage to the ovarian reserve $[20,21]$.

The fact that $\mathrm{AMH}$ expression is restricted to ovarian granulosa cells in women led to establishing AMH levels as a serum marker of granulosa cell tumours (GTCs). Recent studies showed an increased serum AMH concentration in 76-93\% of women with both primary and recurrent GTCs $[22,23]$.

As anti-Müllerian hormone belongs to transforming growth factor- $\beta$ family and its representatives play an important role in ovarian cancer carcinogenesis, the aim of this study was to examine AMH concentration in ovarian cancer patients in relation to clinicopathological features, such as a pathological subtype of the tumor, FIGO stage, grading, and overall 5-year survival.

\section{Patients, Materials, and Methods}

2.1. Patients. We enrolled 72 epithelial ovarian cancer patients aged $45-79$ years (mean $58.5 \pm 10$ years) treated in Department of Gynecology and Obstetrics, Ludwik Rydygier Collegium Medicum in Bydgoszcz in the period of 20052006.

Only women who underwent optimal cytoreductive surgery were considered for further analysis, all of them with residual cancerous focuses smaller than $1 \mathrm{~cm}$ in diameter. All patients were operated by experienced gynecological oncologist. The standard surgical protocol included tumorectomy, hysterectomy with bilateral salpingoovariectomy, pelvic lymphadenectomy, omentectomy, and appendectomy.

Women who had ever used hormonal replacement therapy or had undergone ovulation induction were excluded. All patients were Caucasian, after menopause with BMI range from 19 to $30 \mathrm{~kg} / \mathrm{m}^{2}$.

International Federation of Gynecology and Obstetrics (FIGO) ovarian cancer staging system was used to assess clinical stage of the disease. Early stages were confirmed in 16 patients (FIGO I in 9 women, II in 7) and advanced
TABLE 1: Baseline characteristics of the study group.

\begin{tabular}{lc}
\hline Parameter & $\begin{array}{c}\text { Patients with ovarian cancer } \\
\end{array}$ \\
\hline Age (years) & $58.5 \pm 10.4$ \\
BMI $\left(\mathrm{kg} / \mathrm{m}^{2}\right)$ & $22 \pm 2.2$ \\
FIGO stage $n(\%)$ & \\
I & $9(12.5 \%)$ \\
II & $7(10 \%)$ \\
III & $50(69.5 \%)$ \\
IV & $6(8 \%)$ \\
Histological subtype $n(\%)$ & \\
Serosum & $59(82 \%)$ \\
Mucinous & $5(7 \%)$ \\
Endometrioid & $8(11 \%)$ \\
Histological grading $n(\%)$ & \\
G1 & $9(12.5 \%)$ \\
G2 & $23(32 \%)$ \\
G3 & $40(55.5 \%)$ \\
\hline
\end{tabular}

disease in 56 patients (FIGO III-50, IV-6). All ovarian cancer cases were with histological confirmation, of which 59 (82\%) serous, 5 (7\%) mucinous, and 8 (11\%) endometrioid. Histological examination was performed in the Department of Pathology, Antoni Jurasz University Hospital in Bydgoszcz, and histological grading was determined (G1 in 9, G2 in 23, and G3 in 40 patients).

After optimal cytoreductive surgery, all women underwent 6 courses of chemotherapy based on carboplatin and paclitaxel. Baseline characteristics of the study participants are shown in Table 1.

The Bioethical Committee at the Ludwik Rydygier Collegium Medicum, Nicolaus Copernicus University of Torun, have reviewed and approved this study. All participants have provided informed consent.

2.2. Methods. Blood samples were collected $(10 \mathrm{~mL})$ after admission to the hospital, on the day before surgery. After centrifugation in standard conditions serum was obtained, aliquoted, and stored at $-70^{\circ} \mathrm{C}$ until assayed.

Serum AMH concentration was measured using a twostep sandwich type enzyme immunoassay (Immunotech AMH/MIS ELISA kit, Beckman Coulter). The imprecision of the assay was $12.3 \%$ at $0.2 \mathrm{ng} / \mathrm{mL}$ and $5.1 \%$ at $15.8 \mathrm{ng} / \mathrm{mL}$. The lowest $\mathrm{AMH}$ concentration in a sample which could be detected with a $95 \%$ probability was $0.08 \mathrm{ng} / \mathrm{mL}$ (lower detection limit). Concentration of AMH below $0.14 \mathrm{ng} / \mathrm{mL}$ has been accepted as negative.

Reference values for anti-Müllerian hormone for women over 45 years were less than $1 \mathrm{ng} / \mathrm{mL}$ [24].

The Kolmogorov-Smirnov test was used to assess normality of distribution of investigated parameters. Data were expressed as mean \pm standard deviation and median with 25th-75th percentiles. Comparison between the groups was performed by using the Mann-Whitney $U$ test and the 
TABLE 2: AMH concentration in relation to FIGO stage.

\begin{tabular}{lccccccccc}
\hline & \multirow{2}{*}{ FIGO stage } & \multicolumn{4}{c}{ Statistical characteristics } & \multicolumn{3}{c}{ Mann-Whitney $U$ test } \\
& & Mean & SD & Min & Q1 & Q2 & Q3 & Max & 1.75 \\
AMH/MIS (ng/mL) & I/II & 0.39 & 0.65 & 0.00 & 0.00 & 0.1 & 0.34 & 0.702 \\
& III/IV & 0.34 & 0.79 & 0.00 & 0.00 & 0.08 & 0.33 & 3.26 & \\
\hline
\end{tabular}

AMH/MIS: anti-Müllerian hormone/Müllerian inhibiting substance; FIGO: Federation Internationale de Gynecologie et d'Obstetrique; Max: maximum value; Min: minimum value; Q1: lower quartile; Q2: median; Q3: upper quartile; SD: standard deviation.

TABLE 3: AMH concentration in relation to grading.

\begin{tabular}{ccccccccccc}
\hline & \multirow{2}{*}{ Grading } & \multicolumn{4}{c}{ Statistical characteristics } & \multicolumn{3}{c}{$P$ value Kruskal-Wallis test } \\
& & Mean & SD & Min & Q1 & Q2 & Q3 & Max & 0.58 \\
AMH/MIS (ng/mL) & G1 & 0.17 & 0.21 & 0.00 & 0.00 & 0.1 & 0.34 & 0.875 \\
& G2 & 0.37 & 0.75 & 0.00 & 0.00 & 0.04 & 0.37 & 3.26 & 2.95 \\
\hline
\end{tabular}

AMH/MIS: anti-Müllerian hormone/Müllerian inhibiting substance; Max: maximum value; Min: minimum value; Q1: lower quartile; Q2: median; Q3: upper quartile; SD: standard deviation.

TABLE 4: AMH concentration in relation to histological type of cancer.

\begin{tabular}{lcccccccccc}
\hline & \multirow{2}{*}{ Histological type } & \multicolumn{4}{c}{ Statistical characteristics } & \multicolumn{4}{c}{$P$ value Mann-Whitney $U$ test } \\
& & Mean & SD & Min & Q1 & Q2 & Q3 & Max & \\
\hline \multirow{2}{*}{ AMH/MIS (ng/mL) } & Serosum & 0.23 & 0.49 & 0.00 & 0.00 & 0.04 & 0.3 & 3.26 & 0.653 \\
& Others & 0.68 & 1.04 & 0.00 & 0.00 & 0.02 & 1.44 & 2.95 & \\
\hline
\end{tabular}

AMH/MIS: anti-Müllerian hormone/Müllerian inhibiting substance; Max: maximum value; Min: minimum value; Q1: lower quartile; Q2: median; Q3: upper quartile; SD: standard deviation.

TABle 5: Prognostic factors for overall survival selected by Cox's univariate analysis.

\begin{tabular}{lcccccc}
\hline & & \multicolumn{5}{c}{ Statistical characteristics } \\
& Parameter evaluation & Chi-squared & $P$ value & HR & 95\% CI HR lower endpoint & 95\% CI HR upper endpoint \\
\hline Age & 0,02 & 3,66 & 0,06 & 1,03 & 0,99 & 1,05 \\
AMH (ng/mL) & $-0,01$ & 0,66 & 0,41 & 0,98 & 0,95 & 1,02 \\
Histo-Pat (serosum) & 0,05 & 0,07 & 0,79 & 1,1 & 0,53 & 2,27 \\
Grading (G2 + G3) & $-1,04$ & 4,24 & 0,04 & 0,12 & 0,02 & 0,9 \\
FIGO (III/IV) & $-0,83$ & 10,04 & 0,001 & 0,19 & 0,06 & 0,53 \\
\hline
\end{tabular}

AMH/MIS: anti-Müllerian hormone/Müllerian inhibiting substance; CI: confidence interval; FIGO: Federation Internationale de Gynecologie et d’Obstetrique; HR: hazard ratio.

TABle 6: Prognostic factors for overall survival selected by Cox's multivariate analysis.

\begin{tabular}{lccccc}
\hline & & \multicolumn{2}{c}{ Statistical characteristics } \\
& Parameter evaluation & $P$ value & HR & 95\% CI HR lower endpoint & 95\% CI HR upper endpoint \\
\hline Age & 0,02 & 0,38 & 1,02 & 0,98 & 1,06 \\
AMH (ng/mL) & $-0,02$ & 0,22 & 0,97 & 0,94 & 1,01 \\
Histo-Pat (serosum) & 0,08 & 0,7 & 1,17 & 0,51 & 2,67 \\
Grading (G2 + G3) & $-0,67$ & 0,2 & 0,26 & 0,03 & 2,01 \\
FIGO (III/IV) & $-0,73$ & 0,01 & 0,23 & 0,08 & 0,68 \\
\hline
\end{tabular}

AMH/MIS: anti-Müllerian hormone/Müllerian inhibiting substance; CI: confidence interval; FIGO: Federation Internationale de Gynecologie et d'Obstetrique; HR: hazard ratio. 
Kruskal-Wallis test for non-Gaussian distributed variables. $P$ value $<0.05$ was considered statistically significant.

Overall survival rate was examined for significance using log-rank test and Kaplan-Meier curves.

Univariate and multivariate Cox regressions were performed. For the analysis, a forward selection with a $P$ value of less than 0.05 for entry was applied. The effects of the variables were expressed as hazard ratios per 1 SD change to allow for a better comparability between the effect sizes of the different tested variables.

All statistical analyses were performed using Statistica for Windows Statsoft 10.0.

\section{Results}

In the group of patients with ovarian cancer median serum concentration of AMH was $0.07(0.0-0.37) \mathrm{ng} / \mathrm{mL}$, whereas median concentration in the patients with positive $\mathrm{AMH}$ values $(\geq 0.14 \mathrm{ng} / \mathrm{mL})$ was $0.31(0.15-0.73)$. Values equal to/above $0.14 \mathrm{ng} / \mathrm{mL}$ were found in 44 women $(61 \%)$.

Median concentrations of serum $\mathrm{AMH}$ in relation to FIGO stage did not differ significantly (Table 2 ).

No significant correlations were found between serum AMH concentration and histopathological subtype or grading (Tables 3 and 4).

The results of Cox regression of the predictive power of variables are shown in Tables 5 and 6. In the univariate analysis grading and FIGO were significantly correlated with survival time in women with ovarian cancer. In contrast, in the multivariate analysis only FIGO stage had a statistically significant effect on survival time.

Overall survival rate was also examined in relation to $\mathrm{AMH}$ level. Kaplan-Meier analysis was performed in two groups of ovarian cancer patients: first group $(N=28)$ of women with serum concentration below the detection limit and another $(N=44)$ who displayed values over $0.14 \mathrm{ng} / \mathrm{mL}$ (Figure 1). The long-rank test showed no statistical significance $(P=0.98)$. The analysis of five-year survival rate related to $\mathrm{AMH}$ levels showed no statistically significant differences; there were no differences in survival rates between patients with positive or negative AMH values (Figure 1).

\section{Discussion}

Although it was commonly believed that the cells of epithelial ovarian cancer come from the epithelium covering the surface of the ovary, the current theory states that cancer arises from the cells of the Fallopian tube. Most recent studies indicate that the vast majority of ovarian tumors derive from the fimbriae of Fallopian tube and other components developed from the Müllerian ducts [25]. AntiMüllerian hormone $(\mathrm{AMH})$ initiates the process of regression of Müllerian ducts. Based on these facts some authors suggest that the determination of AMH could be important in the diagnosis and treatment of epithelial ovarian cancer $[26,27]$.

The median concentration of AMH in epithelial ovarian cancer patients was $0.07 \mathrm{ng} / \mathrm{mL}$ taking into account both positive and negative AMH results. However, in the group

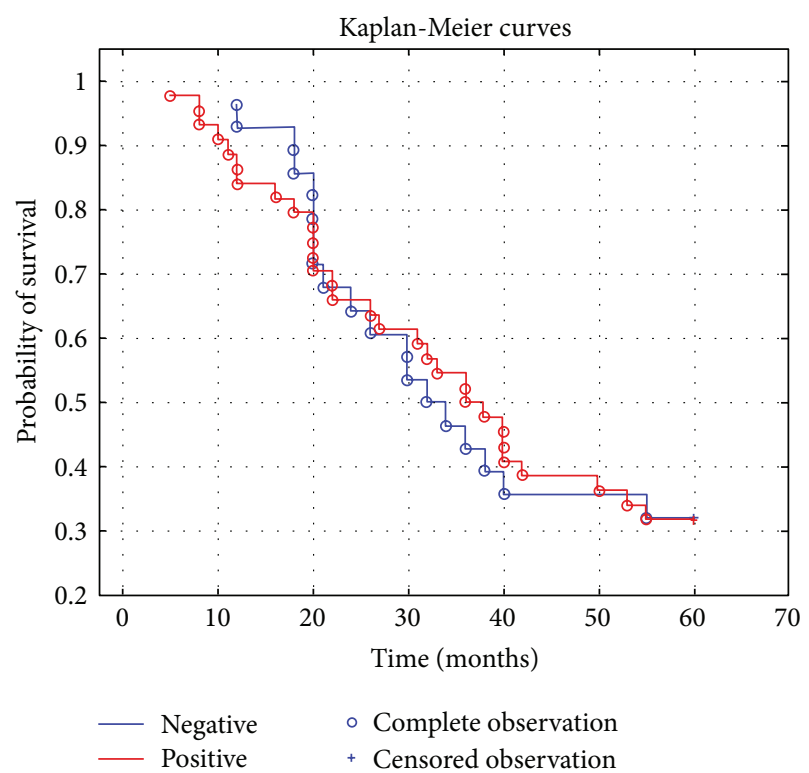

Figure 1: Survival curves in relation to AMH levels.

of women with AMH levels above $0.14 \mathrm{ng} / \mathrm{mL}$, the median was $0.31(0.15-0.73)$. The results observed in our study were similar to those of age-specific AMH values found by others but in healthy women [24].

Despite the lack of statistically significant differences, women with FIGO classifications I and II had lower AMH levels than women with FIGO stages III and IV. On the other hand, according to histological grading, highest $\mathrm{AMH}$ values were observed in women with G1 staging.

Detailed analysis of concentrations of anti-Müllerian hormone has revealed no differences in the levels of $\mathrm{AMH}$, depending on the type of cancer, clinical stage, and histological grade. There was no correlation between serum AMH and the five-year survival rate. There were no differences in years of survival of patients with $\mathrm{AMH}$ in serum compared to patients who were negative for anti-Müllerian hormone. In our study only advanced clinical stage according to FIGO was an independent poor prognostic factor.

To the best of our knowledge there are no available studies demonstrating the usefulness of determination of $\mathrm{AMH}$ concentrations in the serum of patients with epithelial ovarian cancer.

The search for alternative therapies in the treatment of ovarian cancer has led to research on biologically active substances which might inhibit the proliferation of the tumor. Masiakos et al. hypothesized that the anti-Müllerian hormone may serve as such factor because it causes apoptosis and the regression of the Müllerian ducts in embryos, by variation of ovarian tumor cell biology [26]. Further studies showed the presence of type II receptor for AMH in the ovary and in ovarian cancer cells, and its inhibitory effect was confirmed in transgenic mice. In addition, tests were performed on cells obtained from peritoneal fluid from patients with ovarian cancer at FIGO stages III/IV. Masiakos et al. are of the opinion that the determination of $\mathrm{AMH}$ and its receptor by flow 
cytometry could be used in selection of patients with poor prognosis, but this fact has not been confirmed in subsequent studies [26].

In conclusion, AMH belongs to the family of transforming growth factor, which plays an important role in ovarian carcinogenesis. However, none of the limited studies could demonstrate the role of the anti-Müllerian hormone in the serum of patients with ovarian cancer. Similarly, in our study, we failed to show any benefits from the determination of serum AMH in women with ovarian cancer.

Despite our findings concerning epithelial ovarian cancer, serum AMH levels remain well-established marker in granulosa-theca cell tumors. It is necessary to perform further studies to determine the tissue expression of $\mathrm{AMH}$ and type II receptor for $\mathrm{AMH}$ in ovarian cancer tissue and the potential usefulness of monoclonal antibodies against $\mathrm{AMH}$ and its receptor in the diagnosis and treatment of women with ovarian cancer.

\section{References}

[1] R. Siegel, D. Naishadham, and A. Jemal, "Cancer statistics, 2012," CA Cancer Journal for Clinicians, vol. 62, no. 1, pp. 10-29, 2012.

[2] Y. Park, J.-H. Lee, D. J. Hong, E. Y. Lee, and H.-S. Kim, "Diagnostic performances of HE4 and CA125 for the detection of ovarian cancer from patients with various gynecologic and non-gynecologic diseases," Clinical Biochemistry, vol. 44, no. 1011, pp. 884-888, 2011.

[3] M. Walentowicz-Sadlecka, M. Grabiec, P. Sadlecki et al., "Sypniewska G. 25(OH)D3 in patients with ovarian cancer and its correlation with survival," Clinical Biochemistry, vol. 45, pp. 1568-1572, 2012.

[4] A. La Marca, G. Stabile, A. Carducci Artenisio, and A. Volpe, "Serum anti-Mullerian hormone throughout the human menstrual cycle," Human Reproduction, vol. 21, no. 12, pp. 3103-3107, 2006.

[5] M. Š. Alebić, N. Stojanović, and M. Zuvić-Butorac, "The IVF outcome counseling based on the model combining DHEAS and age in patients with low AMH prior to the first cycle of $\mathrm{GnRH}$ antagonist protocol of ovarian stimulation," International Journal of Endocrinology, vol. 2013, Article ID 637919, 2013.

[6] R. Rey and N. Josso, "Regulation of testicular anti-Mullerian hormone secretion," European Journal of Endocrinology, vol. 135, no. 2, pp. 144-152, 1996.

[7] R. R. Behringer, M. J. Finegold, and R. L. Cate, "Müllerianinhibiting substance function during mammalian sexual development," Cell, vol. 79, no. 3, pp. 415-425, 1994.

[8] M. M. Lee, P. K. Donahoe, and T. Hasegawa, "Müllerian inhibiting substance in humans: normal levels from infancy to adulthood," The Journal of Clinical Endocrinology \& Metabolism, vol. 81, no. 2, pp. 571-576, 1996.

[9] M. F. Brougham, P. M. Crofton, E. J. Johnson, N. Evans, R. A. Anderson, and W. H. Wallace, "Anti-Müllerian hormone is a marker of gonadotoxicity in pre- and postpubertal girls treated for cancer: a prospective study," The Journal of Clinical Endocrinology \& Metabolism, vol. 97, pp. 2059-2067, 2012.

[10] C. L. Cook, Y. Siow, S. Taylor, and M. E. Fallat, "Serum mullerian-inhibiting substance levels during normal menstrual cycles," Fertility and Sterility, vol. 73, no. 4, pp. 859-861, 2000.
[11] I. Streuli, T. Fraisse, C. Chapron, G. Bijaoui, P. Bischof, and D. de Ziegler, "Clinical uses of anti-Müllerian hormone assays: pitfalls and promises," Fertility and Sterility, vol. 91, no. 1, pp. 226-230, 2009.

[12] W. M. Baarends, J. T. J. Uilenbroek, P. Kramer et al., "AntiMullerian hormone and anti-Mullerian hormone type II receptor messenger ribonucleic acid expression in rat ovaries during postnatal development, the estrous cycle, and gonadotropininduced follicle growth," Endocrinology, vol. 136, no. 11, pp. 4951-4962, 1995.

[13] J. Shi, Y. Yoshimo, Y. Oshuga et al., "Bone morhogenetic protien6 stimulates gene expression of follicle stimulating hormone receptor, inhibin/activin $\beta$ subunit and anti-Müllerian hormone in human granulose cells," Fertility and Sterility, vol. 92, pp. 1794-1798, 2009.

[14] A. de Vet, J. S. E. Laven, F. H. de Jong, A. P. N. Themmen, and B. C. J. M. Fauser, "Antimüllerian hormone serum levels: a putative marker for ovarian aging," Fertility and Sterility, vol. 77, no. 2, pp. 357-362, 2002.

[15] R. Fanchin, L. M. Schonäuer, C. Righini, J. Guibourdenche, R. Frydman, and J. Taieb, "Serum anti-Müllerian hormone is more strongly related to ovarian follicular status than serum inhibin B, estradiol, FSH and LH on day 3," Human Reproduction, vol. 18, no. 2, pp. 323-327, 2003.

[16] I. A. J. van Rooij, I. den Tonkelaar, F. J. M. Broekmans et al., "Anti-Müllerian hormone is a promising predictor for the occurrence of the menopausal transition," Menopause, vol. 11, no. 6, pp. 601-606, 2004.

[17] A. La Marca, G. Sighinolfi, D. Radi et al., "Anti-Müllerian hormone $(\mathrm{AMH})$ as a predictive marker in assisted reproductive technology (ART)," Human Reproduction Update, vol. 16, no. 2, pp. 113-130, 2009.

[18] S. Broer, B. W. Mol, M. Dolleman, B. Fauser, and J. M. Broekmans, "The role of anti-Müllerian hormone assessment in assisted reproductive technology outcome," Current Opinion in Obstetrics and Gynecology, vol. 22, pp. 193-201, 2010.

[19] G. S. Nakhuda, M. C. Chu, J. G. Wang, M. V. Sauer, and R. A. Lobo, "Elevated serum müllerian-inhibiting substance may be a marker for ovarian hyperstimulation syndrome in normal women undergoing in vitro fertilization," Fertility and Sterility, vol. 85, no. 5, pp. 1541-1543, 2006.

[20] S. Lie Fong, P. J. Lugtenburg, I. Schipper et al., "Anti-müllerian hormone as a marker of ovarian function in women after chemotherapy and radiotherapy for haematological malignancies," Human Reproduction, vol. 23, no. 3, pp. 674-678, 2008.

[21] L. Giuseppe, G. Attilio, D. N. Edoardo, G. Loredana, L. Cristina, and L. Vincenzo, "Ovarian function after cancer treatment in young women affected by Hodgkin disease (HD)," Hematology, vol. 12, no. 2, pp. 141-147, 2007.

[22] R. Rey, J. C. Sabourin, M. Venara et al., "Anti-Müllerian hormone is specific marker of sertoli -and granulose-cell origin in gonadal tumors," Human Pathology, vol. 31, pp. 1202-1203, 2000.

[23] M. L. Gustafson, M. M. Lee, R. E. Scully et al., "Müllerian inhibiting substance as a marker for ovarian sex cord tumor," The New England Journal of Medicine, vol. 326, pp. 1820-1826, 1992.

[24] D. B. Seifer, V. L. Baker, and B. Leader, "Age-specific serum anti-Müllerian hormone values for 17,120 women presenting to fertility centers within the United States," Fertility and Sterility, vol. 95, no. 2, pp. 747-750, 2011. 
[25] L. Dubeau, "The cell of origin of ovarian epithelial tumours," The Lancet Oncology, vol. 9, no. 12, pp. 1191-1197, 2008.

[26] P. T. Masiakos, D. T. MacLaughlin, S. Maheswaran et al., "Human ovarian cancer, cell lines, and primary ascites cells express the human Mullerian inhibiting substance (MIS) type II receptor, bind, and are responsive to MIS," Clinical Cancer Research, vol. 5, no. 11, pp. 3488-3499, 1999.

[27] A. E. Stephen, L. A. Pearsall, B. P. Christian, P. K. Donahoe, J. P. Vacanti, and D. T. MacLaughlin, "Highly purified Müllerian inhibiting substance inhibits human ovarian cancer in vivo," Clinical Cancer Research, vol. 8, no. 8, pp. 2640-2646, 2002. 


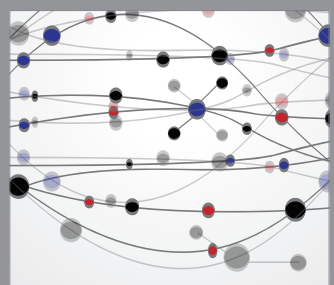

The Scientific World Journal
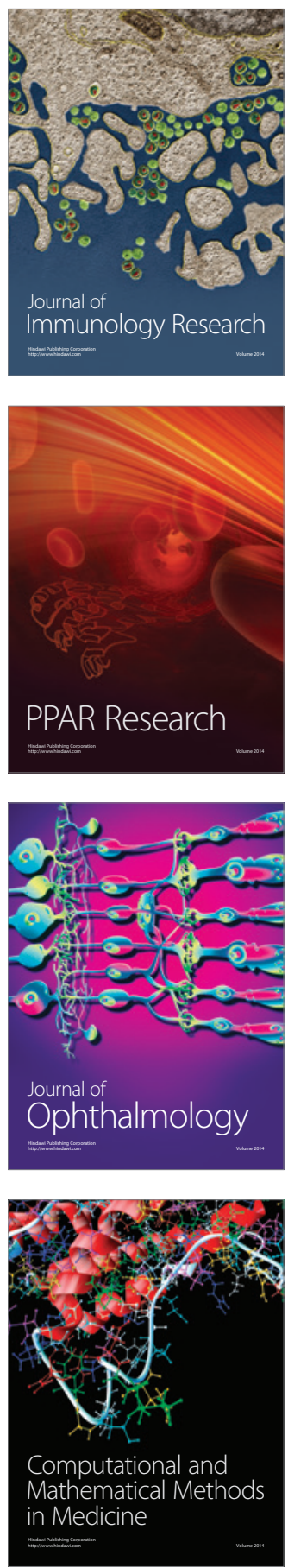

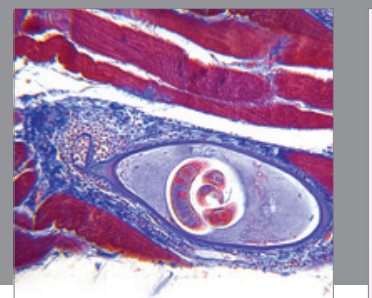

Gastroenterology

Research and Practice
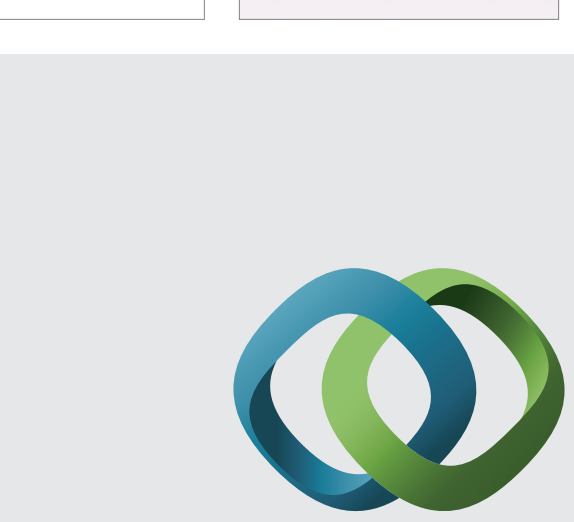

\section{Hindawi}

Submit your manuscripts at

http://www.hindawi.com
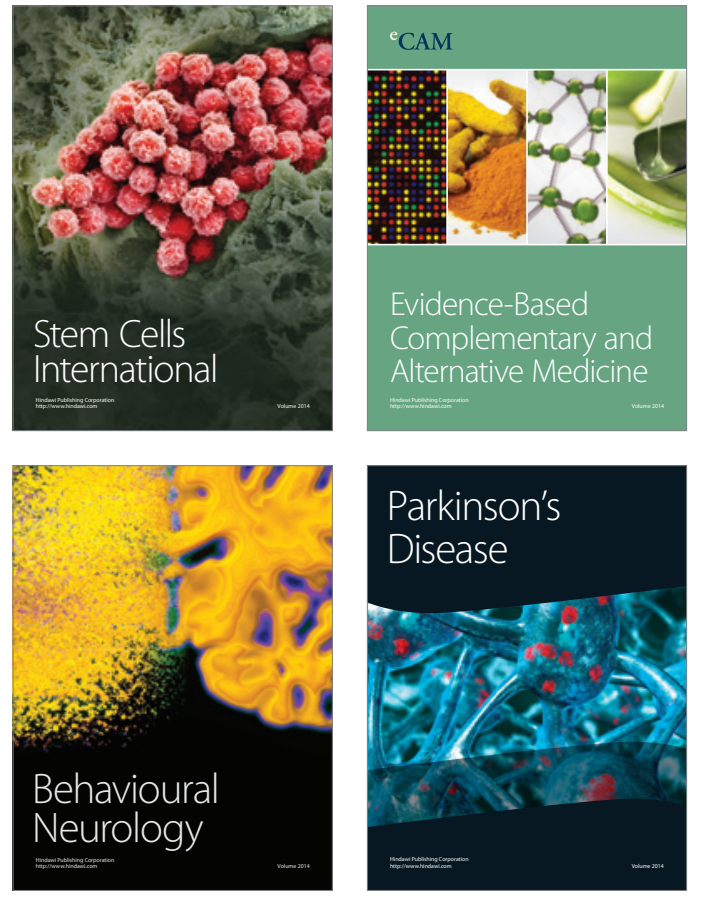
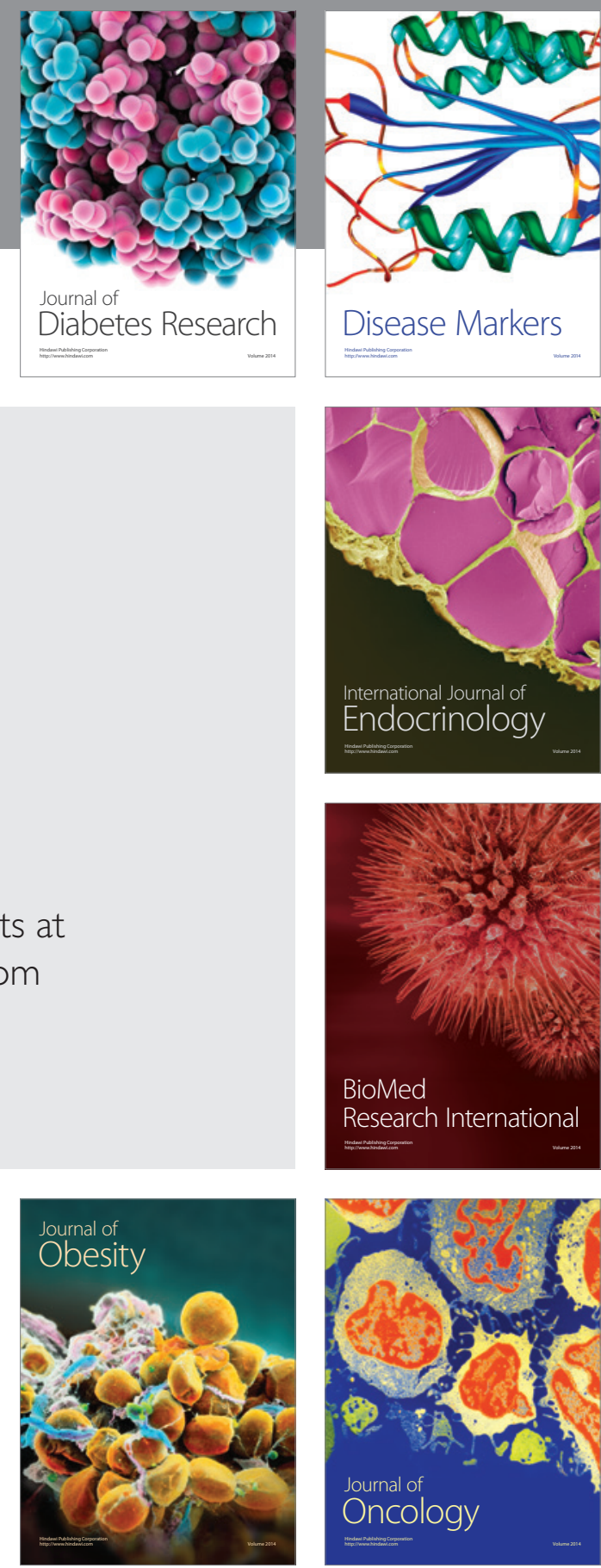

Disease Markers
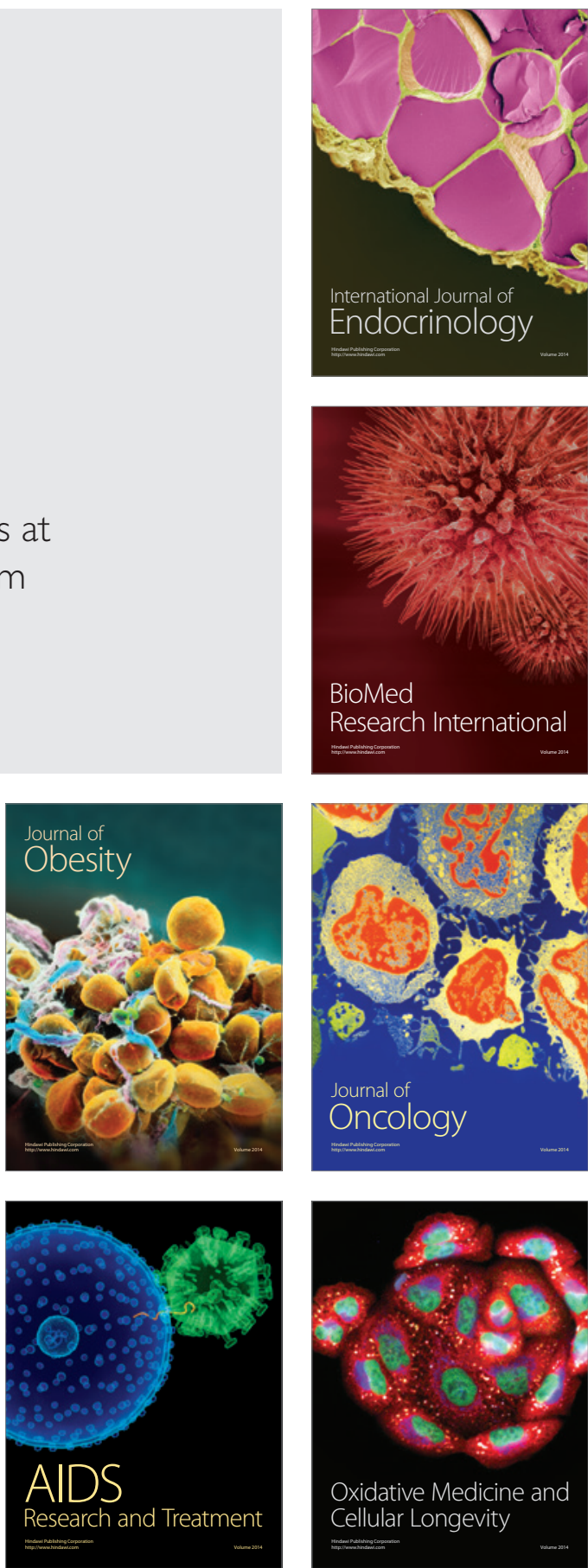